\title{
A importância do cuidado com o pé diabético: ações de prevenção e abordagem clínica
}

The importance of diabetic foot care: preventive care and clinical approach

Carolina Fajardo ${ }^{1}$

\section{RESUMO}

O diabetes é considerado um sério problema de saúde pública, tanto devido ao número de pessoas afetadas quanto às suas complicações, suas incapacitações, além do elevado custo financeiro da sua abordagem terapêutica. Acomete todas as classes socioeconômicas: as populações de países em todos os estágios de desenvolvimento são atingidas por essa enfermidade. Uma das complicações mais temidas ocasionadas pelo diabetes é a perda de um pé ou uma perna. Porém, essa é uma situação que pode ser evitada mediante alguns cuidados. O objetivo desse trabalho é apresentar dados epidemiológicos que evidenciem a importância da prevenção desse problema e aspectos importantes relacionados ao seu manejo clínico, além de questões fundamentais para garantir o controle adequado do diabetes - que são a adesão ao tratamento e o vínculo do paciente com a equipe de saúde.

\section{ABSTRACT}

It is acknowledged that diabetes is a major public health problem, not only due to the large number of individuals that suffer from its complications and incapacitations, but also because of the high cost of the treatment. Diabetes affects the populations of all socio-economical classes in all countries, regardless their stage of development.

One of the most feared diabetes complications is loss of one foot or leg. With some care, however, such situations can be prevented. The purpose of this paper is to present epidemiological data showing clearly the importance of prevention and aspects relevant for clinical care, as well as to make some decisive suggestions for assuring an appropriate control of this chronic disease that are: adherence to treatment and the doctor/patient relation.

PALAVRAS-CHAVE:

- Diabetes Mellitus - prevenção e controle;

- Pé Diabético - tratamento;

- Epidemiologia.

\section{KEY-WORDS:}

- Diabetes Mellitus - prevention and control;

- Diabetic Foot - treatment;

- Epidemiology.

${ }^{1}$ Médica de Família e Comunidade, Residência em MFC/UERJ, médica da Coordenação do PSF/SES-RJ, Rio de Janeiro, Brasil. 


\section{Apresentação e considerações gerais sobre diabetes.}

"A arte de viver

É simplesmente a arte de conviver... Simplesmente, disse eu? Mas como é difícil!"

Mário Quintana

Mário Quintana fala em sua poesia que a arte de viver é simplesmente a arte de conviver, e como isso é difícil por si só. A vida não é nada simples. E a vida com problemas de saúde é mais difícil ainda. Isso, claro, dependendo de como cada um enfrenta o seu problema de saúde. Conviver a vida toda com um problema que exige mudanças nos hábitos de vida e pode ter complicações graves, não sendo uma tarefa simples.

O diabetes é uma doença que transforma a vida das pessoas por ele acometidas, uma vez que exige uma série de mudanças nos hábitos de vida e pode ter sérias complicações. Freqüentemente nos deparamos no nosso dia-a-dia com pessoas com diabetes. É considerado um sério problema de saúde pública, tanto devido ao número de pessoas afetadas quanto às suas complicações, suas incapacitações, além do elevado custo financeiro da sua abordagem terapêutica'. Acomete todas as classes socioeconômicas: as populações de países em todos os estágios de desenvolvimento também são atingidas por essa enfermidade ${ }^{1}$.

Uma das complicações mais temidas ocasionadas pelo diabetes é a perda de um pé ou uma perna. Porém, essa é uma situação que pode ser evitada mediante alguns cuidados. $O$ objetivo desse trabalho é apresentar dados epidemiológicos que evidenciem a importância da prevenção desse problema e aspectos importantes relacio- nados ao seu manejo clínico, além de questões fundamentais para garantir o controle adequado do diabetes - que são a adesão ao tratamento e o vínculo do paciente com a equipe de saúde.

A prevalência do diabetes tipo 2 tem aumentado em proporções epidêmicas, o que é atribuído a vários fatores - como a taxa de urbanização, industrialização, sedentarismo, hábitos alimentares, obesidade, aumento da expectativa de vida - e à maior sobrevida dos diabéticos ${ }^{2}$.

De acordo com a Federação Internacional de Diabetes ${ }^{3}$, há aproximadamente 177 milhões de pessoas com diabetes no mundo, o que corresponde a uma prevalência de $5,2 \%$ nas pessoas adultas (de 20 a 79 anos). Nos países desenvolvidos, o diabetes tipo 2 corresponde a faixa de $85 \%$ a $95 \%$ das pessoas com diabetes, sendo que, nos países em desenvolvimento, provavelmente esse número é maior ${ }^{3}$. Enquanto nos países desenvolvidos a prevalência de diabetes é maior na população mais idosa, nos países em desenvolvimento a prevalência está aumentando na população comparativamente mais jovem, em fase produtiva ${ }^{3}$.

No Brasil, estima-se que haja 5 milhões de pessoas com diabetes, dos quais a metade não sabe que tem a doença ${ }^{2}$. Muitas vezes as pessoas descobrem que tem diabetes por meio das manifestações das suas complicações.

Um grande problema, no Brasil, é o acesso das pessoas com diabetes ao sistema de saúde, o que não possibilita o diagnóstico precoce. Mas, com a política de reorientação da Atenção Básica pela Estratégia de Saúde da Família (ESF), tivemos um grande avanço no acesso ao sistema de saúde. Uma das ações prioritárias da Estratégia é o atendimento ao diabético, além das ações de pro- 
moção e prevenção de saúde.

O diabetes é considerado uma síndrome de etiologia múltipla, decorrente da falta da insulina e/ou incapacidade da insulina exercer adequadamente seus efeitos. Caracteriza-se por uma hiperglicemia crônica que, se não controlada adequadamente, evolui com sérias complicações, como perda da visão, insuficiência renal em estágio terminal, amputação não-traumática dos membros inferiores, infarto agudo do miocárdio, acidente vascular encefálico².

A adesão ao tratamento é fundamental para o melhor controle do diabetes e a redução das suas complicações, mas é difícil de ser alcançada devido à necessidade de tratamento contínuo e prolongado. A abordagem do diabetes é dividida em terapia não-medicamentosa e terapia medicamentosa. As mudanças nos hábitos de vida, como alimentação mais saudável, realização de atividades físicas e interrupção do tabagismo, são elementos essenciais da terapia não-medicamentosa.

Os efeitos colaterais dos remédios e a dificuldade de acesso ao sistema de saúde são outros fatores que influenciam na adesão ao tratamento - a qual deve ser uma meta já na primeira consulta e também nas subseqüentes. Sendo assim, o paciente com diabetes necessita de uma abordagem integral por uma equipe interdisciplinar, a qual trabalha com tecnologia do cuidado, facilitando e promovendo a adesão ao tratamento, e estimula o vínculo do paciente com a equipe de saúde, que é um outro elemento importante para a adesão ao tratamento. Adesão não só ao uso das medicações quando necessárias, mas a um estilo de vida mais saudável.

A classificação utilizada atualmente é a proposta pela Associação Americana de Diabetes (ADA), que se baseia na etiologia do diabetes mellitus ${ }^{4}$. A classificação anterior (proposta pelo National Diabetes Data Group e OMS) tinha por base a forma de tratamento e classificava o diabetes como insulino-dependente e não-insulino-dependente . $^{4}$

Classificação etiológica do diabetes mellitus (Associação Americana de Diabetes)

\section{I- Diabetes Mellitus tipo 1}
A-Auto-imune
B- Idiopático

\section{II- Diabetes Mellitus tipo 2}

\section{III- Outros tipos específicos}
A- Defeitos genéticos da função da celula $\beta$
B- Defeitos genéticos na ação da insulina
C- Doenças do pâncreas exócrino
D- Endocrinopatias
E- Induzida por medicamentos ou produtos químicos
F- Infecções
G- Formas incomuns de diabetes auto- imune
$\mathrm{H}$ - Outras síndromes genéticas às vezes associadas ao diabetes

IV- Diabetes mellitus gestacional 
O diabetes tipo 1 corresponde a faixa de $10 \%$ a $20 \%$ dos casos de diabetes e se caracteriza por uma deficiência absoluta de insulina, que ocorre na maioria das vezes devido a uma destruição auto-imune das células beta. Predomina em crianças e adultos jovens, embora possa surgir em qualquer época da vida. Requer obrigatoriamente o uso de insulina e tem tendência ao quadro de cetoacidose diabética².

O diabetes tipo 2 responde por entre $85 \%$ e $95 \%$ dos casos de diabetes e aparece freqüentemente após os 40 anos de idade. Resulta em geral de graus variáveis de resistência a ação da insulina ou de deficiência de secreção de insulina. A maioria dos pacientes com diabetes tipo 2 (80\%) são obesos e a cetoacidose ocorre apenas em situações especiais como durante as infecções graves $^{2}$. Pode ser controlado apenas com mudanças no estilo de vida, sendo algumas vezes necessário o uso de medicamentos hipoglicemiantes ou antihiperglicemiantes. Nos casos de resistência ao tratamento com essas medicações, pode ser necessário o uso de insulina.

O diagnóstico do diabetes se baseia na história clínica e é confirmado por exames laboratoriais.

Na maioria dos pacientes com diabetes tipo 2, não há sintomas ou estes são inespecíficos, como tontura, dificuldade visual, astenia e/ou cãibras ${ }^{2}$. Os sintomas clássicos de diabetes, como poliúria, polidipsia e polifagia, são bem mais característicos do diabetes tipo 1, embora também possam estar presentes no diabetes tipo $2^{2}$.

A Associação Americana de Diabetes preconiza como critérios para o diagnóstico de diabetes os seguintes valores²:
( Duas glicemias de jejum maior que 126 $\mathrm{mg} / \mathrm{dl}$ em um paciente assintomático ou uma glicemia de jejum = 126 mg/dl em paciente sintomático.

( Glicemia ao acaso $=200 \mathrm{mg} / \mathrm{dl}$, em pacientes com sintomas (poliúria, polidipsia e perda de peso sem explicação).

4 Valor de 2 horas $=200 \mathrm{mg} / \mathrm{dl}$ durante $\mathrm{um}$ teste de tolerância oral à glicose (TOTG).

Essa classificação de diabetes fundamentada na etiologia é a utilizada atualmente pelo Ministério da Saúde ${ }^{5}$.

\section{Incidência e prevalência das complicações e doenças associadas ao diabetes}

No Brasil, não existem dados da população sobre as complicações ou morbidade do diabetes ${ }^{1}$. Portanto, há necessidade de estudos epidemiológicos em relação às complicações do diabetes na população brasileira. Como exceção a essa regra, temos a região metropolitana do Rio de Janeiro, que apresenta a seguinte taxa de incidência de amputação de extremidades: 6,9/100 mil habitantes ${ }^{1}$. Para o conjunto desses casos, a sobrevida acumulada para cinco anos após amputação foi de apenas $55 \%$ para os homens e $59,4 \%$ para as mulheres. Na maioria dos estudos internacionais, essa taxa de amputação equivale a 7,2/100 mil habitantes ${ }^{6}$. Os números mais elevados de amputação foram relatados nas reservas indígenas nos Estados Unidos e os mais baixos em áreas da Dinamarca e Grã Bretanha6. Segundo dados da literatura, porém, há poucos estudos bem realizados relativos a incidência de amputação dos membros inferiores. Há relatos de que, entre 15\% e 19\% dos pacientes diabéticos submetidos a algum tipo 
de amputação, o diabetes foi diagnosticado nesse momento 6 .

Como já relatado, uma das complicações mais temidas ocasionadas pelo diabetes é a perda de um pé ou uma perna. Os problemas com os pés são uma importante causa de morbidade para as pessoas com diabetes, que apresentam um risco 15 vezes maior de serem submetidas a uma amputação do que as pessoas não-diabéticas ${ }^{6}$. As feridas nos pés são uma das principais causas de atendimento hospitalar a essa população.

Estudos mostram que as amputações de pés, pernas e coxas em pacientes com doença arterial periférica e diabetes mellitus aumentaram cinco vezes na cidade do Rio de Janeiro entre 1990 e 2000. Grande parte delas poderia ter sido evitada. A imensa maioria dessas cirurgias $(97,7 \%)$, denominada amputações primárias, foi realizada sem que antes tivesse sido feito o procedimento vascular para restabelecer o fluxo arterial ${ }^{7}$.

Nos Estados Unidos, de 50\% a $70 \%$ de todas as amputações não-traumáticas ocorrem em pessoas diabéticas ${ }^{6}$.

Um dado assustador apresentado em alguns estudos é que apenas $12 \%$ dos médicos examinam os pés dos diabéticos sem estes tenham queixas a apresentar durante a consulta ${ }^{8}$. É sabido que um problema com o pé do diabético pode evoluir para condições mais graves mesmo na ausência de sintomas. Na maioria das pessoas (85\%), as amputações dos membros inferiores relacionadas ao diabetes são precedidas de uma úlcera no pé. A proporção de pacientes que sofre ampuamputação com gangrena varia entre $50 \%$ a $70 \%$ e com infecção, entre $20 \%$ a $50 \%{ }^{6}$.

A prevalência de úlceras nos pés, em países desenvolvidos, corresponde a aproximadamente de $4 \%$ a $10 \%$ dos pacientes com diabetes ${ }^{6}$.

As complicações do pé diabético geram um elevado gasto financeiro com hospitalização prolongada, reabilitação, necessidade de cuidados domiciliares, assistência social, além dos custos indiretos, como o afastamento do trabalho, e emocionais pela perda do pé ou da perna ${ }^{6}$. Estudos mostram que de $40 \%$ a $50 \%$ do custo total para uma doença crônica correspondem aos custos indiretos. O impacto da amputação na qualidade de vida dessas pessoas não tem sido considerado ${ }^{6}$.

Há relatos de que o tempo médio de hospitalização para pacientes com úlceras nos pés varia de 30 a 40 dias, sendo, portanto, 50\% mais prolongado do que nos pacientes sem úlceras nos pés ${ }^{6}$. Outros estudos evidenciaram que os pacientes diabéticos com lesões nos pés e doença vascular permanecem hospitalizados por mais tempo do que as pessoas não-diabéticas com úlcera e doença vascular periférica ${ }^{6}$.

A prevenção das complicações do pé diabético é uma medida de baixo custo, que traz grandes benefícios ${ }^{6}$. Vários estudos apresentaram que uma abordagem multidisciplinar, as ações de prevenção, a terapia educacional e o tratamento multifatorial das úlceras nos pés reduziram as taxas de amputação em torno de $43 \%$ a $85 \%$.

\section{Definição de pé diabético}

Com base nas definições da OMS, o pé diabético é uma condição em que a pessoa com diabetes apresenta infecção, ulceração e ou destruição dos tecidos profundos associadas a anormalidades neurológicas e vários graus de doença vascular periférica nos membros inferiores ${ }^{6}$. 


\section{Fatores de risco do pé diabético}

Embora na maioria dos casos se observe que os fatores de risco para amputação e para desenvolvimento de úlcera sejam semelhantes, de acordo com o Consenso Internacional sobre Pé Diabético $^{6}$, quando se fala em fatores de risco para pé diabético, é preciso diferenciar os fatores relacionados à neuropatia periférica, os relacionados ao desenvolvimento de úlceras nos pés e os relacionados à amputação.

Vários fatores têm sido sugeridos como relacionados ao desenvolvimento de úlceras nos pés, como exemplificado a seguir ${ }^{6}$ : terapêutica precária.

Após o exame clínico do paciente, deve ser feita a sua classificação de risco. O sistema apresentado abaixo é o proposto pelo Consenso sobre o Pé diabético ${ }^{6}$ :

Um estudo realizado para avaliar a efetividade desse sistema de classificação do risco de desenvolver complicações no pé diabético mostrou resultados favoráveis à mesma ${ }^{9}$. Os dados obtidos mostraram que há uma clara tendência de complicações nos pacientes em estágios avançados dessa classificação.

Vários estudos mostraram que o principal

\begin{tabular}{|c|l|l|}
\hline \multicolumn{2}{|c|}{ Sistema de Classificação de Risco } \\
\hline Categoria & \multicolumn{1}{|c|}{ Risco } & Freqüência da avaliação \\
\hline 0 & Neuropatia ausente & Uma vez por ano \\
1 & Neuropatia ausente & Uma vez a cada seis meses \\
\hline 2 & Neuropatia presente, sinais de doença & Uma vez a cada três meses \\
& vascular perférica e/ou deformidades nos pés & \\
\hline 3 & Amputação prévia & Uma vez entre um e três meses \\
\hline
\end{tabular}

\section{IV.1 Úlcera no pé}

૫ Úlcera, amputação prévia.

( Neuropatia: sensitivo-motora.

( Trauma: calçado inadequado; caminhar descalço; quedas, acidentes; objetos no interior dos sapatos.

प Biomecânica

4 Doença Vascular periférica

u Condição socioeconômica: baixa posição social; acesso precário ao sistema de saúde; não-adesão ao tratamento, negligência; educação fator de risco para úlceras nos pés é a presença de neuropatia sensitivo-motora, cuja prevalência estimada varia de $30 \%$ a $70 \%$ das populações estudadas, dependendo das definições e dos critérios diagnósticos utilizados ${ }^{6}$. Em estudos transversais, evidenciou-se que de $80 \%$ a $90 \%$ das úlceras nos pés foram precipitadas por algum trauma externo, como exemplo, o uso de calçados inadequados ${ }^{6}$. 


\section{IV.2 Neuropatia Periférica}

A neuropatia periférica é definida como a presença de sintomas e/ou sinais de disfunção do nervo periférico em pessoas com diabetes após exclusão de outras causas conforme está descrito nas Diretrizes para o Diagnóstico e Abordagem Ambulatorial da Neuropatia Diabética Periférica ${ }^{6}$. A neuropatia periférica pode ser agrupada de acordo com as manifestações clínicas em dois grupos:

u Neuropatia sensitivo-motora é a forma mais comum e a que mais causa problemas nos pés dos diabéticos ${ }^{8}$. Evolui insidiosamente e pode ser assintomática ou causar sintomas importantes, como sensação de queimação e agulhadas nos pés e pernas, que pioram à noite. No exame físico há diminuição da sensibilidade dolorosa, tátil e térmica com distribuição em bota, atrofia da musculatura intrínseca dos pés e ausência dos reflexos aquileus.

4 Neuropatia simpático-periférica - é importante porque causa ressecamento da pele devido à ausência de sudorese, o que leva a pele a ficar rachada e fissurada, facilitando as infecções nos pés. Causa também vasodilatação, propiciando o aparecimento de shunts arteriovenosos, que aumentam o fluxo de sangue na pele, diminuindo, assim, o aporte de nutrientes aos tecidos mais profundos. Portanto, a ausência de sudorese e a distensão das veias dorsais dos pés são evidências de disfunção autonômica que envolve fibras dos nervos simpáticos.

Estudos mostram que a perda da sensibilidade leva a ulceração no pé diabético. Adiminuição da sensibilidade vibratória aumenta sete vezes o risco de desenvolvimento de úlcera nos pés com ou sem isquemia ${ }^{6}$.
A úlcera neuropática é muito mais freqüente do que a úlcera isquêmica.

\section{IV.3 Doença Vascular Periférica}

A doença vascular periférica é o fator mais importante relacionado à evolução de uma úlcera no pé diabético, deve ser diagnosticada por meio do exame clínico dos pés, avaliando cor, temperatura da pele, palpação dos pulsos, medida da pressão do tornozelo ${ }^{6}$. Testes vasculares não-invasivos permitem avaliar a probabilidade de cicatrização de uma úlcera em um pé diabético ${ }^{6}$.

$A$ aterosclerose é mais freqüente nos pacientes diabéticos, nos quais afeta indivíduos mais jovens, com uma progressão mais rápida, multissegmentar e mais distal ${ }^{6}$. A aterosclerose está em geral associada à elevada prevalência de tabagismo, hipertensão e diabetes.

A doença vascular periférica pode ser considerada como sinal da doença aterosclerótica geral. Portanto, deve se buscar doença asterosclerótica em outros lugares como o coração e as caró tidas $^{6}$. O papel da hiperglicemia no desenvolvimenda doença aterosclerótica não está claro. A nefropatia diabética é um marcador para doença vascular generalizada e, provavelmente, os pacientes com nefropatia estão mais suscetíveis ao desenvolvimento de doença vascular periférica ${ }^{6}$.

A doença vascular periférica pode ocorrer mesmo na ausência de sintomas, sendo dividida em quatro estágios, segundo Fontaine ${ }^{6}$ :

Estágio 1: doença arterial oclusiva sem sintomas clínicos.

Estágio 2: claudicação intermitente.

Estágio 3: dor isquêmica em repouso.

Estágio 4: ulceração ou gangrena. 
É preciso ficar atento, pois os pacientes com doença vascular periférica podem apresentar isquemia periférica grave sem sintomas, como conseqüência da perda da sensibilidade devido à neuropatia periférica ${ }^{6}$.

Os pacientes devem ser avaliados em relação à presença de doença vascular periférica pelo menos uma vez por ano, devendo ser pesquisado: história de claudicação intermitente ou dor isquêmica em repouso; palpação dos pulsos das artérias tibiais posteriores e pediosas dorsais; sinais de isquemia crítica - palidez dos pés à elevação, rubor postural, ulceração, necrose da pele ou gangrena 6 .

A investigação vascular não-invasiva deve ser realizada para uma avaliação mais objetiva da perfusão da pele ${ }^{6}$. Os seguintes testes são utilizados:

૫ Pressão do tornozelo.

૫ Pressão do pododáctilo.

प Medidas da pressão de oxigênio transcutâneo.

Esses testes podem ser utilizados para: o diagnóstico e a quantificação da doença vascular periférica; a previsão da cicatrização da lesão em um pé diabético e o seguimento e controle de tratamento ${ }^{6}$. Desses métodos o mais utilizado é a medida da pressão do tornozelo, que pode estar falsamente elevada devido à esclerose média. Nos pacientes com úlcera não-cicatrizada, a doença vascular periférica deve ser investigada, e caso seja necessário realizar uma angiografia.

A revascularização é indicada nas seguintes situações ${ }^{6}$ :

प Baixa probabilidade de cicatrização.

$\checkmark$ Dor isquêmica persistente e em repouso.
4 Claudicação intermitente ameaçando as atividades profissionais do paciente ou limitando seu estilo de vida.

A modificação dos hábitos de vida que representam fatores de risco para o desenvolvimento de claudicação intermitente e amputação deve sempre ser incentivada. O tabagismo, por exemplo, deve ser interrompido uma vez que estudos mostram que as taxas de patência para reconstrução vascular são mais elevadas em pessoas que pararam de fumar6.

A realização de caminhadas é importante, pois foi demonstrado que os programas dessa atividade possibilitam a melhoria da claudicação intermitente em pacientes não-diabéticos. Porém, segundo especialistas, os programas de caminhada não devem ser realizados quando há, nos pacientes, presença de úlceras ou gangrena ${ }^{6}$.

\section{IV.4 Biomecânica}

Os fatores mecânicos (deformidades das articulações, proeminência óssea, calos, mobilidade articular limitada, propriedades dos tecidos alteradas, cirurgia prévia do pé, articulações neuroosteoartropáticas, calçado inadequado, caminhar descalço, quedas, acidentes, objetos no interior dos calçados, grau de atividade) têm um importante papel na etiologia das úlceras nos pés ${ }^{6}$.

O calçado terapêutico é utilizado com o objetivo de aliviar completamente a pressão sobre uma úlcera, que não cicatrizará se a carga mecânica não for removida 6 . Por outro lado, há estudos mais recentes que indicam que, nos pacientes sem deformidades severas nos pés, as ações de cuidado com estes, realizadas tanto pela equipe de saúde quanto pelos pacientes, são mais efetivas do que 
o uso de sapatos terapêuticos ${ }^{10}$.

O calçado protetor é aquele que reduz a pressão nos pés, abaixo do limiar para ulceração, é extremamente importante para prevenir tanto a úlcera inicial quanto à recorrência de úlcera ${ }^{6}$. Os pacientes devem ser orientados a não utilizar novamente um calçado que já provocou ulceração. Estudos mostram que as pessoas que tem um estilo mais ativo de vida apresentam um risco maior de ferimentos nos pés do que aqueles que levam uma vida menos ativa.

Os pacientes devem ser encorajados a utilizar calçados protetores sempre. Os sapatos novos devem ser usados por períodos curtos, inicialmente. Os calçados devem ser trocados várias vezes ao dia e devem ser inspecionados antes do seu uso. Estudos mostram que, quando os calçados protetores são utilizados, há uma prevenção entre $60 \%$ e $85 \%$ de recorrência de úlceras. $A$ adesão ao uso de sapatos protetores é um problema freqüente, provavelmente devido ao custo e por questões estéticas. Um estudo mostrou que o estilo e a aparência do sapato influencia na adesão ao seu uso ${ }^{11}$. Caso a recorrência de ulceração não seja evitada com o uso de calçados apropriados é necessário avaliar a indicação de cirurgia ${ }^{6}$.

\section{IV.5 Úlceras}

Vários fatores influenciam na cicatrização das úlceras nos pacientes com diabetes. Nos centros de excelência as taxas de cicatrização das úlceras estão entre $80 \%$ e $90 \%{ }^{6}$.

É importante reconhecer que a lesão ulcerada é um sinal de uma doença de múltiplos órgãos. A idade é um fator importante em relação à evolução da úlcera. Outros fatores de risco para evolução da lesão ulcerada são: nefropatia diabética; proteinúria; insuficiência renal em estágio terminal; insuficiência cardíaca congestiva; coronariopatias; doença vascular periférica. Portanto, pacientes com úlcera nos pés apresentando comorbidades devem ser tratados incisivamente ${ }^{6}$.

É essencial diferenciar a categoria da úlcera em relação ao fator predisponente, se é a neuropatia ou neuroisquemia. Para facilitar a abordagem das lesões é importante a sua classificação. Dessa forma, o tipo, a localização e a causa de uma úlcera devem ser considerados para auxiliar na decisão da terapêutica apropriada ${ }^{6}$.

O alívio do estresse mecânico é fundamental para a cicatrização da úlcera.

A infecção no pé diabético é uma situação que não pode ser negligenciada e deve ser tratada efetivamente.

As comorbidades associadas devem ser tratadas como o edema da insuficiência cardíaca congestiva, por exemplo, pois influenciam na evolução da lesão ulcerada.

A dor não deve ser subestimada, devendo ser tratada de maneira adequada, dando conforto ao paciente. Muitas vezes, a dor em repouso está relacionada à probabilidade de amputação ${ }^{6}$.

Um bom controle metabólico auxilia no processo de cicatrização da úlcera.

O tratamento tópico é apenas uma parte da abordagem terapêutica das úlceras no pé diabético. A escolha do tratamento tópico é empírica, deve ser fundamentada na condição da úlcera e não implica a cicatrização e não-cicatrização, porém pode alterar a velocidade ${ }^{6}$. 


\section{6 Infecção}

A infecção no pé diabético é uma situação que coloca o paciente em risco para amputação, devendo ser tratada incisivamente. É importante considerar a gravidade das conseqüências da infecção no pé diabético, pois há fatores que contribuem para essa evolução, como a estrutura anatômica, alteração da resposta inflamatória, alterações metabólicas, neuropatia, edema e doença vascular periférica ${ }^{6}$.

É importante ressaltar que sinais e sintomas de infecção, tais como febre, leucocitose e elevação do VHS, podem estar ausentes em pacientes diabéticos com úlceras infectadas nos pés ${ }^{6}$.

A infecção superficial (celulite) é freqüentemente causada por bactérias Gram-positivas, enquanto as infecções mais profundas são causadas por polimicroorganismos, envolvendo as bactérias Gram-negativas e anaeróbicas. Nos casos de infecção profunda e grave do pé, a remoção cirúrgica do tecido infectado é essencial ${ }^{6}$.

A seguinte estratégia deve ser usada na abordagem dos pacientes com lesão ulcerada ${ }^{6}$.

1. Debridamento.

2. Cuidado meticuloso da lesão.

3. Adequado suprimento vascular.

4. Controle metabólico adequado.

5. Antibioticoterapia empírica.

6. Alívio da pressão.

O uso empírico de antibiótico para lesão ulcerada com infecção superficial deve ser ativo contra estafilococos e estreptococos ${ }^{6}$.

No caso de lesões ulceradas profundas, está indicada hospitalização para intervenção cirúrgica e antibioticoterapia de largo espectro ${ }^{6}$.

\section{7 Neuro-osteoartropatia}

Nos pacientes com pé que apresente aumento da temperatura da pele, hiperemia e edema devem se pensar em neuro-osteoartropatia. É importante que seja descartada a possibilidade de infecção para evitar erros de diagnóstico. Essas alterações ósseas e articulares neuropáticas são denominadas pé de Charcot e são consideradas complicações devastadoras do diabetes. Progride rapidamente com fragmentação óssea e destruição das articulações. A osteoartopatia é freqüentemente causada por trauma extrínseco em um pé neuropático. O tratamento dessa complicação é empírico e inclui gesso de contato total e limitação da atividade física ${ }^{6}$.

\section{Abordagem do paciente com pé diabético}

Várias razões apresentadas anteriormente justificam a necessidade do diagnóstico precoce não só do diabetes mellitus como de sua tão temida complicação: o pé diabético. É preciso que o exame minucioso do pé do paciente com diabetes faça parte do exame físico, além de ações educativas que possibilitem ao paciente a realização do autocuidado.

A perda da sensibilidade é o principal fator preditivo do desenvolvimento de úlceras nos pés, por isso o exame neurológico regular dos pés de todos os pacientes diabéticos é fundamental. Todas os profissionais de saúde que trabalham com pessoas com diabetes devem estar habilitados a fazer avaliação neurológica ${ }^{12}$.

No exame do pé do paciente com diabetes, deve se realizar o teste da sensação vibratória utilizando-se um diapasão de $128 \mathrm{~Hz}$, a sensação dolorosa com um pino, apenas quando a pele estiver 
intacta, e a sensação profunda com o martelo (pesquisa do reflexo do tendão de Aquiles). Além dessa avaliação, os testes semiquantitativos podem ser utilizados $^{12}$ :

Monofilamentos de Semmes-Weinstein há estudos que demonstraram que a incapacidade de percepção do monofilamento de $10 \mathrm{~g}$ nos dedos ou dorso do pé prevê futuras ocorrências de úlceras nos pés. São testes simples para realização e de baixo custo.

As seguintes instruções para o uso do monofilamento foram publicadas no manual de hipertensão arterial e diabetes mellitus pelo Ministério da Saúde ${ }^{5}$ :

1- Mostrar o filamento ao paciente e aplicálo em sua mão, para que ele possa reconhecer o tipo de estímulo e "perder o medo".

2- Solicitar que o paciente não olhe para o local em que será efetuado o teste.

3- Pedir para que o paciente preste atenção e simplesmente responda "sim" quando sentir o filamento; não perguntar se ele sente ou não, para não induzi-lo.

4- Ao aplicar o filamento, mantenha-o perpendicularmente à superfície testada, a uma distância de 1 a 2cm: com um movimento suave, façao curvar-se sobre a pele e retire-o, contando mentalmente a seqüência numérica "1001-1002", enquanto o filamento toca a pele, curva-se e sai do contato.

5- Não use movimentos bruscos na aplicação: se o filamento escorregar pelo lado, desconsidere a eventual resposta do paciente e teste o mesmo local, novamente, mais tarde.

6- Use uma seqüência, ao acaso, nos locais de teste, para não induzir o paciente a prever o local seguinte, no qual o filamento será aplicado.
7- Havendo áreas ulceradas, necróticas, cicatrizadas ou hiperceratóticas, teste o perímetro da mesma, e não sobre a lesão.

8- Se o paciente não responder à aplicação do filamento em um determinado local, continue a seqüência randômica e volte posteriormente àquele local, para confirmar.

9- Anote os resultados, segundo a percepção do filamento, em cada região testada.

10- Conserve o filamento protegido, cuidando para não amassá-lo ou quebrá-lo. Se necessário, limpe-o com solução de hipoclorito de sódio a $1: 10$.

Nas figuras 1 e 2, reproduzidas do manual de hipertensão arterial e diabetes mellitus / Ministério da Saúde ${ }^{5}$, são apresentadas as instruções de uso do monofilamento de nylon, que é um bom instrumento para verificar os indivíduos em risco de ulceração:

\section{Figura 1 - Locais de Teste}

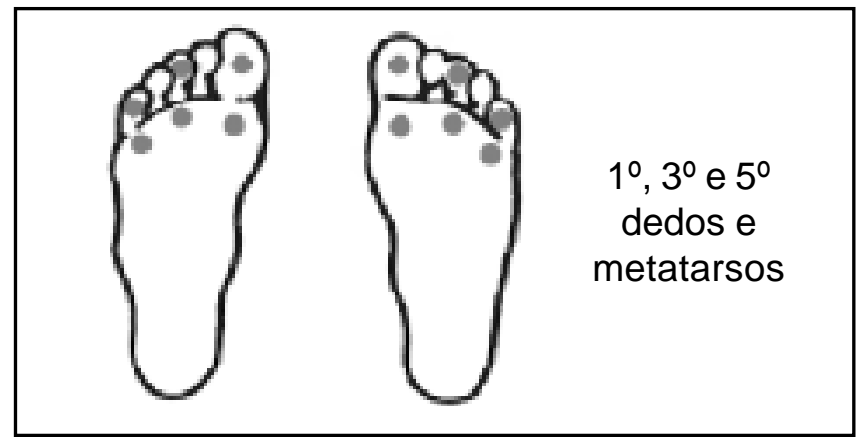

Figura 2 - Aplicação do Monofilamento “A e B"

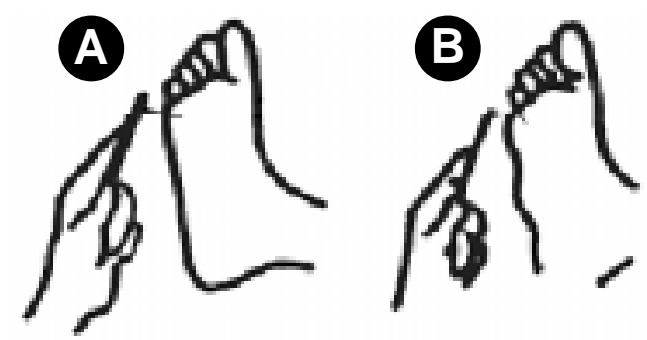


Testes de percepção de vibração - há estudos que demonstraram que a diminuição na sensação vibratória prevê o risco de desenvolvimento de ulceração. Porém, os instrumentos utilizados nesse teste são caros para muitos centros.

Testes quantitativos detalhados de vibração ou de percepção térmica - são utilizados apenas em centros especializados.

De acordo com o Consenso Internacional de Pé Diabético ${ }^{6}$, não há atualmente tratamento farmacológico que produza efeitos importantes na evolução da neuropatia diabética.

O diagnóstico de neuropatia diabética pode ser facilmente realizado em qualquer unidade de saúde com o exame neurológico nos pés. Além disso, há os testes que podem prever o risco de futuras ulcerações. O único tratamento disponível para a neuropatia diabética é o controle metabólico rígido. Apresentada a seguir tabela com as metas do tratamento do diabetes mellitus tipo $2^{13}$.

Quanto ao controle glicêmico, deve-se procurar atingir valores mais próximos do normal. Como muitas vezes não é possível, aceita-se, nestes casos, valores de glicose plasmática em jejum até 126 mg/dl e de duas horas pós-prandial até 160 $\mathrm{mg} / \mathrm{dl}$, além de níveis de glico-hemoglobina até um ponto percentual acima do limite superior do método utilizado. Acima desses valores, é sempre necessário realizar intervenção para melhorar o controle metabólico.

\section{Objetivos do tratamento do diabetes mellitus tipo 2}

\section{Glicose plasmática $(\mathrm{mg} / \mathrm{dl})^{\star}$}

jejum

110

2 horas pós-prandial

140

Glico-hemoglobina (\%)*

Limite superior do método

Coleterol (mg/dl)

total

$<200$

HDL

$>45$

LDL

$<100$

Triglicérides $(\mathrm{mg} / \mathrm{dl})$

$<150$

\section{Pressão arterial $(\mathrm{mm} \mathrm{Hg})$}

Sistólica

Diastólica

$<80$

Índice de massa corporal $(\mathrm{kg} / \mathrm{m} 2)$

$20-25$

Fonte: Consenso Brasileiro de Diabetes. 


\section{Prevenção do Pé Diabético}

Para realizar a prevenção das complicações do pé diabético, as seguintes metas devem ser alcançadas ${ }^{6}$ :

प Realização de exame dos pés pelo menos uma vez ao ano nos pacientes com diabetes e mais freqüentemente naqueles com alto risco de amputação.

( Identificação dos pacientes com alto risco de ulceração.

૫ Educação terapêutica simples, contínua, tanto para os pacientes quanto para os profissionais.

( Uso de calçados adequados.

( Tratamento da patologia não-ulcerativa.

Estudos mostram a redução de ocorrência das lesões nos pés em $50 \%$ dos pacientes que fazem parte de programas de educação terapêutica para cuidados com os pés. Portanto, as ações de educação são importantes nos cuidados dos pacientes com diabetes. O reconhecimento do pé em risco e com lesões em fase inicial é a responsabilidade dos profissionais de saúde, que muitas vezes não é cumprida ${ }^{14}$. Estudos mostram que $50 \%$ dos pacientes submetidos à amputação tinham exame incompleto dos pés. Um outro estudo evidenciou que 22 de 23 amputações abaixo do joelho foram realizadas em pacientes que nunca haviam recebido informações sobre cuidados terapêuticos ou medidas preventivas.

As ações de educação permitem a mudança de atitude do paciente, estimulando-o ao autocuidado e possibilitando uma maior adesão ao tratamento proposto. O paciente precisa participar ativamente do tratamento, pois muitas vezes ele pode identificar problemas em fase inicial em seu próprio pé, tomando assim as providências necessárias e procurando auxílio profissional mais cedo. Há poucos dados na literatura sobre as técnicas educativas utilizadas ${ }^{6}$. Contudo, estudos mostram que, quando se utiliza a técnica do aprendizado participativo, o resultado é o paciente com uma atitude mais apropriada quanto aos cuidados com os pés e uma redução das demandas de pés requerendo tratamento.

Os pacientes com diabetes ou os seus cuidadores devem estar capacitados aos seguintes cuidados com seus pés ${ }^{7}$ :

1- Inspeção dos pés todos os dias.

- Usando um espelho, se necessário, para visualizar todo pé. Isso deve ser feito em uma área com iluminação apropriada.

- Buscando qualquer sinal de pressão (vermelhidão, bolhas, calosidades), cortes ou fendas entre os dedos, feridas ou qualquer mudança na cor habitual de seus pés.

- Atenção para qualquer mudança na temperatura da pele dos seus pés.

2 - Lavar os seus pés todos os dias.

- Usando um sabão suave e água morna (nunca quente).

- Enxugando seus pés com uma toalha macia, sem esfregar a pele.

- Prestando muita atenção ao enxugar a pele entre os dedos.

3 - Manter a pele de seus pés macia e flexível.

- Aplicando uma loção hidratante sobre os pés secos, exceto entre os dedos ou sobre feridas abertas ou rachaduras.

- Não aplicando talco em seus pés, pois isso poderá produzir ressecamento. 
4 - Usar, todos os dias, meias limpas e sapatos resistentes e bem adaptados.

5 - Prestar atenção especial aos cuidados das unhas.

- Ao lixar as unhas, usar sempre uma lixa apropriada e respeitar o formato delas.

- Ao cortar as unhas, dispor de boa iluminação. Se o paciente não consegue enxergar bem, ele deve pedir a alguém que corte suas unhas. Cortar as unhas imediatamente após lavar os pés, quando elas estão macias. Usar tesouras com pontas arredondadas. Não usar tesouras para unhas com pontas encurvadas. Respeitar o formato da unha.

- Não cortar calosidades nem unhas encravadas.

Para que tenham os pés sadios, os pacientes devem ser orientados a:

- Usar sempre sapatos e meias - até mesmo em casa. Chinelos resistentes são apropriados para serem usados em casa, devem ser calçados logo após sair da cama. Nunca devem andar descalços.

- Usar meias limpas - as brancas sem costura são as melhores. Evitar as meias apertadas.

- Sacudir os sapatos antes de calçá-los.

- Nunca cruzar as pernas quando estiver sentado ou deitado; ao cruzá-las a circulação será afetada.

- Realizar exercícios suficientes - caminhar é bom. Se o paciente precisa ficar sentado por longos períodos de tempo, deve ser orientado a inclinar os pés para cima e para baixo, movendo os dedos com freqüência.

- Levantar os pés sobre um banquinho ou cadeira enquanto estiver sentado.
- Não ficar andando sem rumo no escuro o paciente deve acender as luzes antes de entrar em um quarto escuro ou de subir escadas.

- Não fumar! O fumo reduz a circulação sangüínea e, para os pacientes que sofrem de diabetes, isso pode provocar a perda de um membro.

Além dessas orientações o paciente deve saber que, ao detectar um dos sintomas abaixo, ele deve avisar o seu médico imediatamente:

- Uma ferida aberta ou uma bolha em seus pés.

- Pernas frias e dormentes com uma coloração pálida ou azulada.

- Dores, tipo câimbras, em suas pernas ao caminhar.

- Pé de atleta (coceira, bolhas, pele descamada) entre seus dedos ou na sola dos pés.

Os pacientes precisam de um canal de comunicação com a equipe de saúde que cuida deles. Eles devem ter acesso para a comunicação imediata sobre alterações nos seus pés, o que permitirá o tratamento eficaz.

\section{Conclusão}

Após a apresentação de todos esses dados, os seguintes aspectos devem ser ressaltados para garantir um atendimento adequado ao paciente diabético:

( A importância das ações de promoção de saúde e prevenção do diabetes mellitus e suas complicações, em evidência nesse trabalho o pé diabético.

(4) A necessidade de garantir o acesso a todos os níveis de atenção à saúde.

(- A valorização de uma abordagem humanizada e interdisciplinar na assistência ao paciente 
diabético.

( A importância da realização do exame minucioso dos pés dos pacientes com diabetes, na maioria das vezes negligenciada pelos profissionais de saúde.

( A valorização da adesão ao tratamento e do vínculo do paciente com a equipe de saúde como estratégias essenciais para que se alcance os resultados esperados.

( A realização de atividades educativas que trabalhem com os profissionais de saúde e os pacientes, as questões relacionadas ao cuidado dos pés e a necessidade de ter uma vida com hábitos mais saudáveis.

\section{Referências}

1. Franco JF. Epidemiologia do diabetes mellitus. In: Lessa I. O adulto brasileiro e as doenças da modernidade - epidemiologia das doenças crônicas não transmissíveis. São Paulo - Rio de Janeiro: HUCITEC/ABRASCO, 1998. p. 123-37.

2. Teixeira L, Machado AC. Diabetes Mellitus Novos Critérios de Classificação e Diagnóstico. In: Endocrinologia Clínica. $1^{\text {a }}$ edição.Rio de Janeiro: MEDSI; 1999. p. 353-62.

3. Internacional Diabetes Federation. Disponível em: <http://www.idf.org>. Acesso em 25 nov. 2003. 4. Vilar L, Canadas V. Diabetes Mellitus. In: Filgueira NA, Costa Júnior JI, Leitão CCS, Lucena VG, Melo HRL, Brito CAA. Condutas em Clínica Médica. $2^{\text {a }}$ ed. Rio de Janeiro: MEDSI; 2001. p. 465-94.

5. Brasil. Ministério da Saúde. Plano de reorganização da atenção básica à hipertensão arterial e ao diabetes mellitus: manual de hipertensão arterial e diabetes mellitus. Brasília, DF: Departamento de Ações Programáticas Estratégicas; 2001.
6. Grupo de Trabalho sobre Pé Diabético.Consenso Internacional sobre Pé Diabético/ publicado sob a direção de Hermelinda Cordeiro Pedrosa; tradução de Ana Cláudia de Andrade, Hermelinda Cordeiro Pedrosa. Brasília, DF: Secretaria de Estado de Saúde do Distrito Federal; 2001.

7. Sociedade Brasileira de Diabetes. Disponível em: $<$ http://www.diabetes.org.br>. Acesso em: 25 nov. 2003.

8. Macedo G. Conduta no pé diabético. In: Endocrinologia Clínica. $1^{\text {a }}$ ed. Rio de Janeiro: MEDSI; 1999. p. 484-89.

9. Peters EJG, Lavery LA. Effectiveness of the Diabetic Foot Risk Classification System of the International Working Group on the Diabetic Foot. Diabetes Care. aug. 2001; 24(8): 1442-47.

10. Reiber GE, Smith DG, Wallace C, Sullivan K, Hayes S, Vath C, Maciejewski ML, Yu O, Heagerty PJ, LeMaster J. Effect of Therapeutic Footwear on Foot Reulceration in Patients With Diabetes. JAMA 2002; 287: 2552-58.

11. Macfarlane DJ, Jensen JL. Factors in Diabetic Footwear Compliance. J Am Pediatr Med Assoc 2003; 93(6): 485-91.

12. American Diabetes Association. Preventive Foot Care in People with Diabetes. Diabetes Care. jan. 2002; 25(1) 569-70.

13. Sociedade Brasileira de Diabetes. Consenso Brasileiro sobre Diabetes. Diagnóstico e Classificação do Diabetes Mellitus e Tratamento do Diabetes MellitusTipo 2. Maio. 2000.

14. Levin ME. Management of the Diabetic Foot: Preventing Amputation. South Med J. 2002; 95(1):10-20. 


\section{Endereço para correspondência:}

Carolina Fajardo

Rua Conde de Baependi, número 70/402.

CEP 22231-140 - Rio de Janeiro - RJ

\section{Endereço eletrônico:}

carolfajardo@yahoo.com.br 